\title{
Decay of Correlations in Fermi Systems at Non-zero Temperature
}

\author{
M. B. Hastings \\ T-13 and Center for Nonlinear Studies, Los Alamos National Laboratory, Los Alamos, NM 87545, hastings@lanl.gov
}

(June 7, 2004)

\begin{abstract}
The locality of correlation functions is considered for Fermi systems at non-zero temperature. We show that for all short-range, lattice Hamiltonians, the correlation function of any two fermionic operators decays exponentially with a correlation length which is of order the inverse temperature for small temperature. We discuss applications to numerical simulation of quantum systems at non-zero temperature.
\end{abstract}

PACS: 71.10.Fd,31.15.Ew,05.30.-d

The locality of correlation functions is a fundamental property of a quantum system, with great practical importance in density functional theory simulation of quantum systems [1]: if the correlation functions are shortranged, then one can develop fast methods $O(N)$ for simulating a system by solving it in local regions $[2,3]$. How, though, can we know a priori that the correlation functions will be short-ranged? For quantum systems, it was proven recently [4] that the exponential decay in space of connected correlation functions follows from the existence of a gap between the ground and first excited states. If instead the gap vanishes, as occurs at a quantum phase transition [5], then long-range correlations may occur at zero temperature, while slow fluctuations of the order parameters may give rise to exotic physical properties.

However, another important possibility in which certain correlations will decay exponentially in space is to consider a system at non-zero temperature. In this paper, we prove that correlation functions of fermionic operators are short-ranged at non-zero temperature, and we show that the correlation length is bounded, for small temperature, by a quantity which scales inversely with the temperature. The results are general, and apply to any lattice Hamiltonian $\mathcal{H}$ with finite range $R$ as defined below. There are no periodicity requirements and no requirements of free or weakly interacting particles.

Previously, it had been suggested that for noninteracting particles the correlation length would be inversely proportional to the square-root of the temperature [6]. However, later it was argued that, for certain specific systems with free particles in periodic potentials, the correlation length may be much marger, scaling inversely with the temperature [7]. Thus, the results in [7] provide an example showing in this paper we have, in fact, derived the best possible bound on the scaling of the correlation length with temperature for small temperature.

It is important that we consider fermionic operators; in contrast, bosonic operators may develop long-range order at non-zero temperature. To define what is meant by fermionic and bosonic operators, we first define, for each lattice site, various fermionic and bosonic operators which act only on that site. Possible examples of fermionic operators on a site $i$ include electron creation and annihilation operators, $\psi_{i}^{\dagger}, \psi_{i}$; examples of bosonic operators may include spin operators, phonon operators, etc... The defining property of an operator on a single site being fermionic or bosonic is that operators on different sites anticommute with each other if they are both fermionic, while they commute with each other if at least one of the operators is bosonic. In general, we define an operator $A$ to be fermionic if $A$ can be written as a sum of products of single-site operators, where each product includes an odd number of fermionic single-site operators. Thus, $\psi_{i}$ and $\psi_{i} \psi_{j}^{\dagger} \psi_{k}$ are all allowed fermionic operators.

We define the correlation function at inverse temperature $\beta$ by $\langle A B\rangle_{\beta} \equiv Z^{-1} \operatorname{Tr}[A B \exp (-\beta H)]$, where $Z=\operatorname{Tr}[\exp (-\beta H)]$. The trace is taken in the grand canonical ensemble. Then, we show that if $A$ and $B$ are fermionic operators separated by distance $l$ then

$$
\langle A B\rangle_{\beta} \leq c\||A\|\|| \mid B\| \exp (-l / \xi),
$$

where $c$ is a constant and, for large $\beta$, the correlation length $\xi$ is of order $v \beta$, with $v$ a characteristic velocity of the system. Here, we define the distance $l$ between operators to be the shortest distance between any pair of sites $i, j$ where some operator on site $i$ appears in $A$ and some operator on $j$ appears in $B$.

The finite range condition is defined following $[4,9]$ : we require that we can write $\mathcal{H}=\sum_{i} \mathcal{H}_{i}$, where $i$ ranges over lattice sites, and where the commutator $\left[\mathcal{H}_{i}, O\right]=0$ for any operator $O$ which only acts on sites $j$ which are more than a distance $R$ from site $i$, and where, for some constant $J,\left\|\mathcal{H}_{i}\right\| \leq J$ for all $i$. These conditions are sufficient to enforce the requirement of a finite group velocity below. As an example of the finite range, a hopping term $\psi_{i}^{\dagger} \psi_{j}$ in the Hamiltonian $\mathcal{H}_{i}$ is allowed if site $j$ is within range $R$ of site $i$.

To prove Eq. (1), we first express the correlation function in terms of anti-commutators using an integral representation. Then, we bound the anti-commutators using the finite group velocity [8].

Integral Representation of Correlation Function - Let $A$ have matrix elements $A_{i j}$ in a basis of eigenvectors of $\mathcal{H}$, where states $i, j$ have energies $E_{i}, E_{j}$ respectively. Let $\left(A_{\omega}\right)_{i j}=A_{i j} \delta\left(E_{i}-E_{j}-\omega\right)$. Note that $A=\int \mathrm{d} \omega A_{\omega}$. 
The expectation value $\left\langle A_{\omega} B\right\rangle_{\beta}=Z^{-1} \sum_{i, j} \delta\left(E_{i}-\right.$ $\left.E_{j}-\omega\right) A_{i j} B_{j i} \exp \left(-\beta E_{i}\right)$. Similarly, $\left\langle B A_{\omega}\right\rangle_{\beta}=$ $Z^{-1} \sum_{i, j} \delta\left(E_{i}-E_{j}-\omega\right) B_{j i} A_{i j} \exp \left(-\beta E_{j}\right)$. However, $\delta\left(E_{i}-E_{j}-\omega\right) \exp \left(-\beta E_{j}\right)=\delta\left(E_{i}-E_{j}-\omega\right) \exp \left(-\beta E_{i}\right)$ $\exp (\beta \omega)$. Thus, $\left\langle B A_{\omega}\right\rangle_{\beta}=\left\langle A_{\omega} B\right\rangle_{\beta} \exp (\beta \omega)$. Therefore,

$$
\left\langle A_{\omega} B\right\rangle_{\beta}=\frac{1}{1+\exp (\beta \omega)}\left\langle\left\{A_{\omega}, B\right\}\right\rangle_{\beta} .
$$

It was essential to use the grand canonical ensemble to derive this relation: if instead we worked in the canonical ensemble with a fixed number, $N$, of particles, then in the definition of $\left\langle A_{\omega} B\right\rangle_{\beta}$ as a sum over states $i, j$ we would require that state $i$ had $N$ particles while in the definition of $\left\langle B A_{\omega}\right\rangle_{\beta}$ we would instead require that state $j$ had $N$ particles. If the operator $A$ changes the number of particles we would not be able to relate these two expectation values as simply as was done above.

Next, we use $(1+\exp (\beta \omega))^{-1}=1 / 2-\beta^{-1} \sum_{\text {nodd }}(\omega-$ $i n \pi / \beta)^{-1}$, where the sum ranges over all positive and negative odd $n$. For $n>0$, we have $(\omega-i n \pi / \beta)^{-1}=$ $i \int_{0}^{\infty} \mathrm{d} t \exp [-(i \omega+n \pi / \beta) t]$. Similarly, for $n<0$, we have $(\omega-i n \pi / \beta)^{-1}=-i \int_{0}^{\infty} \mathrm{d} t \exp [(i \omega+n \pi / \beta) t]$. Thus,

$$
\frac{i}{\beta} \sum_{n \text { odd }, n>0} \int_{0}^{\infty} \mathrm{d} t \exp (-n \pi t / \beta)(\exp (i \omega t)-\exp (-i \omega t)),
$$

where now the sum ranges only over positive, odd $n$.

Now, we define the time evolution of operators by $A(t)=\exp (i \mathcal{H} t) A \exp (-i \mathcal{H} t)$. Thus, $A_{\omega}(t)=$ $\exp (i \omega t) A_{\omega}$. Combining Eqs. $(2,3)$, we get

$$
\begin{array}{r}
\left\langle A_{\omega} B\right\rangle_{\beta}=1 / 2\left\langle\left\{A_{\omega}, B\right\}\right\rangle_{\beta}+ \\
\frac{i}{\beta} \int_{0}^{\infty} \mathrm{d} t \frac{\exp (-\pi t / \beta)}{1-\exp (-2 \pi t / \beta)}\left\langle\left\{A_{\omega}(t)-A_{\omega}(-t), B\right\}\right\rangle_{\beta},
\end{array}
$$

where we have used $\sum_{\text {nodd }, n>0} \exp (-n \pi t / \beta)=$ $\exp (-\pi t / \beta) /(1-\exp (-2 \pi t / \beta))$. Finally, we integrate Eq. (4) over $\omega$ to get

$$
\begin{array}{r}
\langle A B\rangle_{\beta}=1 / 2\langle\{A, B\}\rangle_{\beta}+ \\
\frac{i}{\beta} \int_{0}^{\infty} \mathrm{d} t \frac{\exp (-\pi t / \beta)}{1-\exp (-2 \pi t / \beta)}\langle\{A(t)-A(-t), B\}\rangle_{\beta} .
\end{array}
$$

Finite Group Velocity - In $[9,4]$, it was proven that if we have a Hamiltonian obeying the finite range condition above, then for any two bosonic operators $A, B$, separated in space by a distance $l$, one can bound the operator norm of the commutator: $\|[A(t), B]\| \leq\|A\|\|B\| \sum_{j} g\left(t, l_{j}\right)$, where the sum ranges over sites $j$ which appear in operator $B$ and $l_{j}$ is the distance from $j$ to the closest site $i$ in the operator $A$. Here $g\left(t, l_{j}\right)$ is a function with the following properties: (1) $g$ is symmetric in $t$ so that $g(t, l)=g(-t, l) ;(2) g(t, l) \leq\left(t / t^{\prime}\right) g\left(t^{\prime}, l\right)$ for $t<t^{\prime}$ and $t, t^{\prime}>0$; and (3) there exists a constant $c_{1}$ such that $g\left(c_{1} l, l\right)$ is exponentially decaying in $l$ for large $l$ with some correlation length $\xi_{C}$. Defining $v=c_{1}^{-1}$, we can view $v$ as a characteristic velocity of the system.

While in $[9,4]$ only bosonic operators were considered, for fermionic operators, a similar bound can be proven: $\|\{A(t), B\}\| \leq\|A\|\|B\| \sum_{j} g\left(t, l_{j}\right)$. This proof can be proven following the same steps as in [4] with commutators replaced by anti-commutators throughout.

We now combine the bound on anti-commutators with Eq. (5). The physics is as follows: Eq. (4) gives the correlation function as an integral over time. However, for times $t$ large compared to $\beta$, the integral is cut off by the exponential; however, for times $t$ of order $\beta$ or less, the anti-commutator of Eq. (4) is small for $l$ large compared to $v \beta$.

More precisely, note that since $A, B$ are separated by a distance $l$, then $\{A, B\}=0$. Thus,

$$
\left|\langle A B\rangle_{\beta}\right| \leq
$$

$$
\begin{aligned}
& \left|\frac{i}{\beta} \int_{0}^{c_{1} l} \mathrm{~d} t \frac{\exp (-\pi t / \beta)}{1-\exp (-2 \pi t / \beta)}\langle\{A(t)-A(-t), B\}\rangle_{\beta}\right|+ \\
& \quad\left|\frac{i}{\beta} \int_{c_{1} l}^{\infty} \mathrm{d} t \frac{\exp (-\pi t / \beta)}{1-\exp (-2 \pi t / \beta)}\langle\{A(t)-A(-t), B\}\rangle_{\beta}\right| .
\end{aligned}
$$

Note that $\left|\langle\{A(t)-A(-t), B\}\rangle_{\beta}\right| \leq 4\|A\||| \mid B \|$. Thus, the integral over times $t>c_{1} l$ in Eq. (6) is bounded in absolute value by $4\|A\|\|B\| \beta^{-1} \int_{c_{1} l}^{\infty} \exp (-\pi t / \beta) /(1-$ $\exp (-2 \pi t / \beta)) \leq 4\|A\|\|B\| \pi^{-1} \exp \left(-\pi c_{1} l / \beta\right) /(1-$ $\left.\exp \left(-2 \pi c_{1} l / \beta\right)\right)$.

For

times $t<c_{1} l$, we use the bound on the anti-commutator: $\left|\langle\{A(t), B\}\rangle_{\beta}\right| \leq\|\{A(t), B\}\| \leq\left[t /\left(c_{1} l\right)\right] \sum_{j} g\left(c_{1} l, l_{j}\right)$. Also, $\exp (-\pi t / \beta) /(1-\exp (-2 \pi t / \beta)) \leq \beta /(2 \pi t)$. Thus, the integral over times $t<c_{1} l$ in Eq. (6) is bounded in absolute value by $\|A\|\|B\| \sum_{j} g\left(c_{1} l, l_{j}\right) \int_{0}^{c_{1} l} \mathrm{~d} t\left(2 \pi c_{1} l\right)^{-1}=$ $(2 \pi)^{-1}\|A\|\|B\| \sum_{j} g\left(c_{1} l, l_{j}\right)$.

Therefore,

$$
\left|\langle A B\rangle_{\omega}\right| \leq\|A\|\|B\|\left(\frac{\sum_{j} g\left(c_{1} l, l_{j}\right)}{2 \pi}+\frac{4}{\pi} \frac{\exp \left(-\pi c_{1} l / \beta\right)}{1-\exp \left(-2 \pi c_{1} l / \beta\right)}\right) .
$$

Since $l_{j} \geq l, g\left(c_{1} l, l_{j}\right)$ decays exponentially for large $l$ as $\exp \left(-l / \xi_{C}\right)$. Also, $\exp \left(-\pi c_{1} l / \beta\right)$ decays exponentially with correlation length $v \beta / \pi$. Thus, the correlation length $\xi$ is bounded by the maximum of $\xi_{C}$ and $v \beta / \pi$, so that for small temperature $\xi \leq v \beta / \pi$. Thus, Eq. (1) follows.

It is interesting to compare Eq. (7) to the result found in [4] for systems in the ground state with a gap $\Delta E$ between the ground and first excited states. There, the resulting correlation length was the maximum of $\xi_{C}$ and $2 v / \Delta E$.

Importance of Lattice Structure - This bound was derived for systems with a lattice structure. To illustrate 
the importance of a lattice, consider free fermions on a lattice in one dimension, with Hamiltonian $t \psi_{i}^{\dagger} \psi_{i+1}+$ h.c. The Fermi velocity depends on filling, and is maximum at half-filling. Thus, the Fermi velocity can be bounded, and is at most of order $t$. Now, consider free fermions in free space one dimension, with Hamiltonian $\psi^{\dagger}(x)\left[\partial^{2} /(2 m)\right] \psi(x)$. The Fermi velocity again depends on the particle density, but can be increased without limit as the particle density is increased.

This is the physical reason why, for systems which are not on a lattice, one cannot (without knowing more about the density and other details of the system) bound the anti-commutator. One cannot, from the Hamiltonian alone, provide a velocity $v$ such that the anti-commutator $\{A(t), B\}$ is small for $t<l / v$.

Discussion - We have proven a bound on the correlation of fermionic operators on non-zero temperature, Eq. (7). There are specific examples [7] which show that this bound on the scaling of the correlation length with temperature is the best possible (although the prefactor $v / \pi$ in the correlation length $\xi=v \beta / \pi$ might not be the best possible). Similarly, while it had been shown that for periodic, non-interacting insulators in one-dimension the correlation length scales inversely with the squareroot of the gap [10], there are again specific examples of non-interacting systems [7] which show that the bound [4] on the scaling of the correlation length with the gap is also the best possible. The case of the canonical, as opposed to grand canonical, ensemble is an interesting future problem.

In addition to the basic interest in this result in quantum statistical mechanics, this result is of importance in quantum simulation using density functional theory. Also, the integral representation, Eq. (5) may prove useful as a means of computing the density matrix for free fermions at non-zero temperature; for these systems, if $A(0)$ is the fermionic creation operator on a single site and $B$ is a fermionic annihilation operator, then $A(t)$ is a sum of fermionic creation operators and the anticommutator is trivial to compute. This is a matter for future research.

Acknowledgements - I thank A. Niklasson for useful discussions. This work was supported by DOE contract W-7405-ENG-36.

[1] W. Kohn, Phys. Rev. Lett. 76, 3168 (1996).

[2] W. Yang, Phys. Rev. Lett. 66, 1438 (1991).

[3] S. Goedecker and M. Teter, Phys. Rev. B 51, 9455 (1995).

[4] M. B. Hastings, Phys. Rev. B 69104431 (2004).

[5] S. Sachdev, Quantum Phase Transitions, (Cambridge University Press, New York, 1999).

[6] R. Baer and M. Head-Gordon, Phys. Rev. Lett. 79, 3962 (1997).

[7] S. Ismail-Beigi and T. A. Arias, Phys. Rev. Lett. 82, 2127 (1999).
[8] M. B. Hastings, preprint cond-mat/0405587.

[9] E. Lieb and D. Robinson, Commun. Math. Phys. 28, 251 (1972).

[10] W. Kohn, Phys. Rev. 115, 809 (1959). 